ISSN: 2386-3919 - e-ISSN: 2386-3927

DOI: https://doi.org/10.14201/et20203812948

\title{
DESARROLLO CURRICULAR DE LAS COMPETENCIAS CLAVE: SU EVALUACIÓN PARA EL APRENDIZAJE DESDE LA NORMATIVA EDUCATIVA
}

\author{
Curricular Development of Key Competencies: \\ Their Assessment for Learning from the Educational Laws
}

\author{
María Antonia Manassero-Mas y Ángel VÁzQueZ-Alonso
}

Recibido: 18/03/2020; Aceptado: 14/07/2020; Publicado: 30/12/2020

Ref. Bibl. MARÍA ANTONIA MANASSERO-MAS y ÁNGEL VÁZQUEZ-ALONSO. Desarrollo curricular de las competencias clave: su evaluación para el aprendizaje desde la normativa educativa. Enseñanza E Teaching, 38, 1-2020, 29-48.

RESUMEN: La ley española de educación (2006) introdujo las competencias clave como elementos del currículo escolar; su diseño innovador, pero excesivamente genérico y abierto, estancó el desarrollo del currículo basado en competencias y, especialmente, la evaluación competencial; estas dificultades han motivado desatención a la orientación competencial en las aulas y la investigación educativa. Algunas tendencias y normativa educativa posterior han propuesto nuevas orientaciones, cuya aplicación y desarrollo pueden permitir afrontar esas dificultades de la enseñanza y la evaluación de los aprendizajes basados en competencias y que este artículo pretende desarrollar. Para ello, se realiza un análisis del contenido del currículo para una materia (Física y Química), centrado en los estándares y resultados de aprendizaje evaluables, para desarrollar y construir el perfil competencial de esta materia; el análisis se complementa con la elaboración de una rúbrica, diseñada para ejemplificar la evaluación de los estándares del perfil competencial. Ambos instrumentos, perfil y rúbricas, se proponen como modelos prácticos para guiar una enseñanza y una evaluación competenciales y formativas en aulas e investigación. Finalmente, se discuten las posibilidades y limitaciones de este modelo para evaluar competencias formativamente, como desarrollo ajustado a la normativa, y, a la vez, práctico y viable para el profesorado. 
MARÍA ANTONIA MANASSERO-MAS Y ÁNGEL VÁZQUEZ-ALONSO

DESARROLLO CURRICULAR DE LAS COMPETENCIAS CLAVE: SU EVALUACIÓN PARA EL APRENDIZAJE DESDE LA NORMATIVA EDUCATIVA

Palabras clave: competencias básicas; evaluación basada en competencias; perfiles competenciales de materias; estándares y resultados de aprendizaje evaluables; competencias básicas en ciencia y tecnología.

SUMMARY: The 2006 Spanish educational law introduced key competencies as elements of the Spanish school curriculum; its innovative design, but excessively generic and open, stalled the development of the competency-based curriculum and, especially, the assessment of competency. The difficulties on these developments caused some neglect key competences in the classrooms and in educational research. Some trends and subsequent educational regulations have proposed new orientations, whose application and development may allow facing the difficulties in teaching and assessing competency-based learning that this article aims to develop. To this aim, a curriculum content analysis of a specific subject (Physics and Chemistry) is carried out to develop and build the competence profile of this subject, focused on the assessment learning standards; the analysis is complemented by the elaboration of a rubric, which is designed to exemplify the evaluation of the competency profile standards. Both instruments, subject profiles and rubrics, are proposed as practical models for teachers to guide a competency-based teaching and assessment for learning. Finally, the possibilities and limitations of this model for competency assessment for learning are discussed, as a development in tune to the regulations, and, at the same time, practical and viable for teachers.

Key words: key competences; formative assessment; competence profiles of subjects; measurable learning standards and results; basic competences in science and technology.

\section{INTRODUCCIÓN}

El concepto de competencia surgió en el contexto laboral, para ajustar las capacidades de cada trabajador a las demandas específicas del puesto de trabajo. En los últimos lustros, los acelerados cambios propios de las globalizadas y multiculturales sociedades del conocimiento y la información han proyectado la competencia sobre las políticas educativas, universalizando el objetivo de educar por competencias, cuyo fin es lograr resultados de aprendizaje funcionales, es decir, desarrollar capacidades de realizar eficazmente tareas y demandas complejas y cambiantes a lo largo de toda la vida. Esta innovación ha generado una catarata de literatura educativa sobre el tema que la limitación de espacio aquí solo permite resumir. El objetivo de este estudio es analizar las posibilidades de educar y evaluar competencialmente y proponer un modelo para ello, aplicando los elementos del marco normativo actual. 
MARÍA ANTONIA MANASSERO-MAS Y ÁNGEL VÁZQUEZ-ALONSO

DESARROLLO CURRICULAR DE LAS COMPETENCIAS CLAVE: SU EVALUACIÓN PARA EL APRENDIZAJE DESDE LA NORMATIVA EDUCATIVA

\section{El MARCO TEÓRICO DE LAS COMPETENCIAS}

La competencia es un constructo complejo, cuya definición varía según países, estudios, grados, niveles o autores. Cano (2008) cita una veintena de definiciones diferentes de competencia en el ámbito educativo, puntualizando que lo esencial es la movilización múltiple y contextualizada de conocimientos, destrezas y actitudes para la acción. Desde la perspectiva psicológica también se enfatizan las características personales (motivos, rasgos de personalidad, auto

concepto, valores, etc.) y las características del comportamiento observable de la competencia, que representaría el lazo de unión entre las características individuales y las cualidades generales (Pereda y Berrocal, 2001). Por ello, varios proyectos desarrollan marcos conceptuales que definen e identifican las competencias necesarias para desarrollar una vida personal y socialmente valiosa en las modernas y plurales sociedades de la información.

La UNESCO (Delors, 1996) estableció los principios precedentes de una enseñanza basada en competencias al identificar los pilares básicos de la educación permanente para el siglo XXI, consistentes en «aprender a conocer» (conocimientos), "aprender a hacer» (procesos), "aprender a ser» (carácter) y "aprender a convivir» (ciudadanía).

El proyecto DeSeCo (OECD, 2002) seleccionó las competencias clave, consideradas esenciales para la vida personal y social y definió la competencia como:

la capacidad de responder a demandas complejas y llevar a cabo tareas diversas de forma adecuada. ... supone una combinación de habilidades prácticas, conocimientos, motivación, valores éticos, actitudes, emociones, y otros componentes sociales y de comportamiento que se movilizan conjuntamente para lograr una acción eficaz (Eurydice, 2002: 13).

La Recomendación 2006/962/EC del Parlamento Europeo y del Consejo (Unión Europea, 2006) insta a los Estados miembros a desarrollar la oferta de competencias clave para el aprendizaje permanente. Define competencia como una combinación de conocimientos, capacidades o destrezas y actitudes adecuadas al contexto y considera claves aquellas competencias necesarias para el desarrollo y la realización personales, la ciudadanía activa, la inclusión social y el empleo en el siglo XxI, y esenciales para el bienestar, el crecimiento económico y la innovación de las sociedades europeas. El marco europeo contempla ocho competencias clave (Tabla 1) y siete temas transversales que combinan conocimientos, destrezas y actitudes esenciales vinculadas a todas las competencias.

Por su relegación respecto a las competencias, se considera importante subrayar aquí los siguientes siete temas transversales: resolución de problemas, evaluación de riesgos, iniciativa, toma de decisiones, gestión positiva de las emociones, pensamiento crítico y creatividad. En la práctica, como se expone en el marco jurídico, estos temas transversales están incluidos en las competencias. Desde la perspectiva conceptual, esa heterogénea lista de temas transversales puede 
MARÍA ANTONIA MANASSERO-MAS Y ÁNGEL VÁZQUEZ-ALONSO

DESARROLLO CURRICULAR DE LAS COMPETENCIAS CLAVE: SU EVALUACIÓN PARA EL APRENDIZAJE DESDE LA NORMATIVA EDUCATIVA

simplificarse, pues, en el marco de la psicología cognitiva, la mayoría de los temas transversales citados se identifican con las denominadas destrezas de pensamiento de alto nivel, también denominadas por otros autores pensamiento crítico. Por ello, el pensamiento crítico, como tema transversal, podría ser el constructo más general de todos los temas transversales, ya que, conceptualmente, englobaría al resto de los temas transversales como destrezas constitutivas del pensamiento crítico (Fisher, 2009; Manassero y Vázquez, 2019).

\section{TABLA 1}

Las competencias claves definidas por las autoridades europeas (ocho)

y españolas (siete) de los resultados de los aprendizajes educativos

\begin{tabular}{|c|c|}
\hline $\begin{array}{l}\text { RECOMENDACIÓN 2006/962/CE } \\
\text { PARLAMENTO Y CONSEJO EUROPEOS }\end{array}$ & $\begin{array}{l}\text { REAL DECRETO } 1105 / 2014 \\
\text { (MECD, ESPAÑA) }\end{array}$ \\
\hline $\begin{array}{l}\text { Comunicación en lengua materna. } \\
\text { Comunicación en lenguas extranjeras. }\end{array}$ & Comunicación lingüística. \\
\hline $\begin{array}{l}\text { Competencia matemática y competencias } \\
\text { básicas en ciencia y tecnología (СВСут). }\end{array}$ & $\begin{array}{c}\text { Competencia matemática y competencias } \\
\text { básicas en ciencia y tecnología. }\end{array}$ \\
\hline Competencia digital (TIC). & Competencia digital. \\
\hline Aprender a aprender. & Aprender a aprender. \\
\hline Competencias sociales y cívicas. & Competencias sociales y cívicas. \\
\hline $\begin{array}{l}\text { Sentido de la iniciativa y } \\
\text { espíritu de empresa. }\end{array}$ & $\begin{array}{l}\text { Sentido de iniciativa y } \\
\text { espíritu emprendedor. }\end{array}$ \\
\hline Conciencia y expresiones culturales. & Conciencia y expresiones culturales. \\
\hline
\end{tabular}

El Espacio Europeo de Educación Superior plantea la necesidad de capacitar a los ciudadanos para hacer frente a los acelerados cambios derivados de la globalización y del desarrollo tecnológico y, para ello, el proceso de Convergencia Europea incorpora las sugerencias del proyecto Tuning, que proponen una formación superior basada en competencias, para garantizar la empleabilidad y la profesionalidad. Las competencias Tuning son una combinación dinámica de conocimiento, comprensión, capacidades y habilidades, que conforman los puntos de referencia compartidos para el diseño (describir objetivos) y la evaluación de los planes de estudios (evaluables) y que también permiten describir el grado de capacidad de una persona para desempeñarlos (desarrollables). En los diferentes países se observan también formulaciones diversas de competencias clave generales y específicas para la educación superior, que constituyen variaciones sobre denominaciones similares a las contenidas en la Tabla 1 y los temas transversales. 
El programa PISA (Programa para la Evaluación Internacional de Estudiantes), un hito en la evaluación de competencias, parte de la hipótesis de que el éxito de un estudiante de 15 años consiste en la adquisición de un conjunto de competencias funcionales para su vida en tres áreas diana (lingüística, matemática y científica). Específicamente para la última, el marco teórico PISA 2015 (OECD, 2013) plantea la alfabetización científica como «todo lo que deberían conocer, valorar y ser capaces de hacer como preparación para la vida en sociedad, participar en un discurso razonado sobre la ciencia y la tecnología y tomar decisiones de la vida diaria».

La esencia de una competencia, pues, consiste en integrar en la acción múltiples saberes: saber (cognitivo), saber hacer (destrezas) y saber ser y estar (valores y actitudes). Los conocimientos integrados son aquellos relevantes para la resolución de problemas (complejidad), bajo un principio de funcionalidad (organizar y reorganizar lo aprendido y transferir su aplicación a situaciones prácticas nuevas, imprevisibles o relevantes, personal o socialmente). Ser competente implica saber, poder, querer y saber hacer, ser y estar (componentes de la competencia) en situaciones concretas y diferentes contextos del ámbito personal, social o laboral; a la vez, representa la capacidad de integración creativa, flexible y responsable de conocimientos, habilidades, creencias, valores y actitudes exigidos por la ejecución de una tarea, y cuyo logro demuestra la expresión más alta de transferencia de conocimientos y aprendizajes a contextos diversos (VV. AA., 2013).

La competencia se configura también como un conocimiento en la práctica, es decir, un conocimiento desarrollado también a través de la participación en prácticas reales, de modo que la ejecución práctica de las competencias dota a las personas de autonomía, la cual les permite continuar aprendiendo y actuando eficazmente a lo largo de toda la vida. Por ello, las competencias se pueden desarrollar tanto en contextos formales, a través del currículo educativo, como en los contextos educativos no formales e informales.

Desde una perspectiva crítica se ha destacado también la proyección innovadora de las competencias para lograr aprendizajes relevantes y funcionales, caracterizados por la integración dinámica de elementos múltiples y la transferibilidad y aplicabilidad de los aprendizajes a otros contextos (diferentes al académico o escolar). Esta cualidad configura un importante criterio de evaluación en el aprendizaje de competencias, caracterizado por la capacidad de transferencia de los resultados de aprendizaje; por ello, en su rol de diseñador de actividades de aprendizaje orientadas al dominio competencial, el profesorado debe aplicar dosis de realismo y también cierta interdisciplinariedad. Sin olvidar el riesgo de quedarse en una mera reforma estructural, que no llegue a innovar los modos de enseñar y aprender profundamente, el aprendizaje por competencias requiere una trasformación innovadora (inspirada por actividades auténticas) del diseño curricular, las metodologías de enseñanza y la evaluación de los aprendizajes (Bolívar, 2008). Además, la formación del profesorado resulta una necesidad obvia y una condición clave para que la innovación implicada en la educación por competencias sea real y exitosa (Valle y Manso, 2013). 
MARÍA ANTONIA MANASSERO-MAS Y ÁNGEL VÁZQUEZ-ALONSO

DESARROLLO CURRICULAR DE LAS COMPETENCIAS CLAVE: SU EVALUACIÓN PARA EL APRENDIZAJE DESDE LA NORMATIVA EDUCATIVA

\section{LA EVALUACIÓN DE LAS COMPETENCIAS}

Atendiendo al viejo heurístico educativo de que «lo que no se evalúa no cuenta" (probablemente no se aprende), es fácil intuir que la evaluación por y de las competencias es un elemento clave de su desarrollo curricular. Como toda evaluación, la evaluación competencial tiene dos referencias básicas: el marco teórico general de la evaluación educativa y el marco específico atribuido a las propias competencias, como objetos de evaluación.

El marco teórico de la evaluación educativa está bien establecido en numerosos manuales, por lo que sus principios no se tratan aquí exhaustivamente. Baste recordar que evaluar consiste en emitir un juicio de valor sobre el objeto que se evalúa (en este caso, las competencias); esto implica definir bien el objeto, definir bien los indicadores de evaluación, emplear métodos e instrumentos adecuados para la recogida de datos y definir los criterios para interpretar datos y elaborar el juicio final de valor (De la Orden, 2011). La realidad de la evaluación educativa actual es que debe superar algunos obstáculos en forma de mitos y lugares comunes del profesorado acerca de la evaluación, tales como identificar evaluación con examen o calificación; ello requiere integrar la evaluación en la enseñanza, en lugar de separarla, sustituyendo progresivamente la dominancia de la evaluación sumativa con más evaluación para el aprendizaje y complementando la mera calificación con la amplificación de la función formativa y orientadora de la evaluación, de modo que los estudiantes puedan continuar aprendiendo toda su vida (OECD, 2008a).

Las recomendaciones de Looney y Michel (2014) para ayudar a los profesores en la evaluación de competencias van en la línea de desarrollar marcos de evaluación coherentes, que pasan por el uso de nuevas herramientas y criterios, tanto sumativos (portfolio y TIC) como formativos (cuestiones, entrevistas, auto- y coevaluación, realimentación y orientación para mejorar) y otras múltiples medidas que permitan tener una visión global del progreso de los aprendices en la comprensión de relaciones, procesos de razonamiento y destrezas complejas (en lugar de solo medir el recuerdo de fragmentos de conocimientos).

El diseño y desarrollo de un currículo por competencias implica que las tareas de aprendizaje y evaluación deben integrar los diversos componentes curriculares de la competencia (conocimientos, procedimientos, actitudes, valores, etc.) en ejecuciones eficaces en un contexto determinado. Esto significa que la evaluación debe ser auténtica (evaluar ejecuciones observables) donde la adaptación al contexto, el desarrollo progresivo de la competencia y la autoreflexión en la ejecución deben ser indicadores importantes de la competencia. Con estas características, los instrumentos de evaluación centrados en conocimientos (tests, pruebas objetivas, ejercicios mecánicos, reproducciones memorísticas, etc.) deberán abrirse a las nuevas finalidades y lógicas (ser competentes), que requieren nuevas estrategias y herramientas adaptadas a ellas (escalas, listas de chequeo, rúbricas, evaluación de progreso, portafolios, diarios, apuntes, trabajos, proyectos, prácticas, etc.). 
MARÍA ANTONIA MANASSERO-MAS Y ÁNGEL VÁZQUEZ-ALONSO DESDE LA NORMATIVA EDUCATIVA

La red europea sobre competencias clave (KeyCoNet) realizó un informe sobre el estado de la aplicación de las competencias clave en 29 países europeos (Looney y Michel, 2014). Por su interés para este estudio, se refleja la recomendación referida al desarrollo de marcos de evaluación coherentes, que propone nuevas herramientas y criterios para ayudar al profesorado a evaluar las competencias, tanto en evaluaciones sumativas como formativas. Las evaluaciones formativas para el aula proponen la interacción y orientación para mejorar, la indagación, los diálogos abiertos, la autoevaluación y la evaluación inter pares. Los enfoques innovadores de la evaluación sumativa sugieren evaluar mediante portafolios o TIC, que permitan documentar habilidades más complejas y visualizar procesos de razonamiento.

Una investigación sobre los procesos de cambio educativo en España analizó el proceso de incorporar las competencias en la práctica educativa y sus resultados ofrecen un panorama desolador, especialmente para la evaluación (Monarca y Rappoport, 2013). Los profesores encuestados señalan que la evaluación es el tema menos claro sobre competencias y trasladan a la evaluación el dualismo curricular competencias/contenidos, concluyendo que se puede evaluar por contenidos o por competencias. El resultado es que la evaluación sigue estando enfocada en los contenidos conceptuales (y en su mera repetición o explicación).

En general, la mayoría de estudios e iniciativas sobre competencias desarrollados en la última década en España se centran en su enseñanza/aprendizaje, relegando su evaluación. Entre las excepciones, De la Orden (2011) reflexiona sobre la necesidad de un sistema de evaluación válido para competencias, partiendo de dos modelos: competencia como desempeño exitoso o como integración de saberes. El autor se decanta por la evaluación de ejecuciones, centrado en las evidencias que confieran validez al juicio de evaluación de competencias a través del cumplimiento de criterios generales de validez de las evaluaciones: representatividad de muestras, ajuste, relevancia, generalización y carácter formativo de las tareas de competencias (extraer consecuencias para optimizar la enseñanza por competencias).

Recuperando la heurística inicial de este apartado (lo que no se evalúa no cuenta) se puede concluir que la ausencia del tema de la evaluación de las competencias en la investigación educativa puede ser otro indicador negativo más acerca de la escasa relevancia de la educación por competencias. Por ello, se revisan los marcos normativos de la evaluación de competencias y de la competencia científica de PISA, como marcos teóricos adicionales sobre la aplicación de este estudio en asignaturas de ciencias.

\section{El MaRCo JURÍDiCO-LEGAL DE LAS COMPETENCIAS}

Siguiendo las recomendaciones de la UE, España incorporó las competencias clave a la educación obligatoria con el nombre de competencias básicas (Ley Orgánica de Educación 2/2006, de 3 de mayo) y que, desde una perspectiva disciplinar para el área de ciencias, fueron divulgadas por Cañas, Martín y Nieda (2007). La 
MARÍA ANTONIA MANASSERO-MAS Y ÁNGEL VÁZQUEZ-ALONSO

DESARROLLO CURRICULAR DE LAS COMPETENCIAS CLAVE: SU EVALUACIÓN PARA EL APRENDIZAJE DESDE LA NORMATIVA EDUCATIVA

Ley Orgánica para la Mejora de Calidad Educativa 8/2013, de 9 de diciembre, puso el énfasis en la relación del currículo basado en competencias para el desarrollo del talento de todos los estudiantes (Marina, 2010).

Las habilidades cognitivas son parte esencial del talento, aunque no sean los componentes únicos ni suficientes, y, por ello, es necesario adquirirlas desde edades tempranas en la escuela. Hoy, estas habilidades se formulan en términos de destrezas transversales, entre las cuales son frecuentes las alusiones a pensamiento crítico, trabajo en equipo, gestión de la diversidad, creatividad, capacidad de comunicar o iniciativa, que también incluyen actitudes transversales, tales como autoconfianza, implicación, entusiasmo, esfuerzo, constancia, afrontamiento del cambio o fracaso, etc. (Unión Europea, 2006). La Unión Europea propone la adquisición de las competencias como objetivo general de la educación, lo cual supone una simplificación, pero también un cambio radical del desarrollo curricular, para lograr una visión más interdisciplinar, personalizada, efectiva y sólida de los aprendizajes, en paralelo con una mayor autonomía docente. Siguiendo estas directrices, el currículo escolar español actual regula los siguientes elementos: competencias (Tabla 1), objetivos, contenidos (conocimientos, habilidades, destrezas y actitudes), metodología didáctica, criterios de evaluación y estándares y resultados de aprendizaje evaluables (ERAE). El currículo define las competencias como capacidades para aplicar integradamente los contenidos propios de cada enseñanza y etapa educativa, con el fin de lograr la eficaz y adecuada realización de actividades y resolución de problemas complejos; los criterios de evaluación y los estándares y resultados de aprendizaje evaluables (ERAE) son los elementos que concretan la evaluación del grado de adquisición de las competencias y del logro de los objetivos de cada enseñanza o etapa.

Dado que el proceso de enseñanza-aprendizaje basado en competencias se caracteriza por la transversalidad (interdisciplinariedad y transferencia a diversos contextos), el dinamismo (desarrollo de mejores niveles de desempeño con la práctica progresiva) y el carácter integral (formación integral de las personas), el aprendizaje competencial debe abordarse desde todas las áreas de conocimiento (humanidades, sociales, ciencias, matemáticas y artes) y desde las diversas instancias educativas (formales, no formales e informales) para descubrir nuevas formas de acción y habilidades, que, satisfaciendo las condiciones anteriores, produzcan el desarrollo de la capacidad de aprendizaje a lo largo de toda la vida.

Con el fin de facilitar la adecuada inserción de las competencias en el currículo escolar, la normativa reciente ha descrito las relaciones entre competencias, contenidos y criterios de evaluación en una Orden (ECD/65/2015, de 21 de enero) con rango de norma básica (aplicable en todo el territorio español). La norma describe las finalidades y aspectos distintivos de las competencias (Tabla 1) y establece que todas las asignaturas del currículo deben contribuir al desarrollo y logro por los estudiantes de las competencias desde su ámbito propio. La integración de las competencias clave en los currículos de las asignaturas se define, se explicita y se desarrolla a través de los estándares y resultados de aprendizaje (ERAE), y este principio normativo se aplicará en este estudio para elaborar la relación entre competencias y ERAE como 
MARÍA ANTONIA MANASSERO-MAS Y ÁNGEL VÁZQUEZ-ALONSO

DESARROLLO CURRICULAR DE LAS COMPETENCIAS CLAVE: SU EVALUACIÓN PARA EL APRENDIZAJE

DESDE LA NORMATIVA EDUCATIVA

la base que permite proyectar la evaluación educativa centrada en competencias y rigurosamente dentro del marco normativo.

\subsection{La evaluación de las competencias en el marco educativo jurídico-legal}

Respecto a la evaluación de las competencias en cada asignatura, la norma mencionada (Orden ECD/65/2015) ofrece algunas pautas clarificadoras e imperativas (el énfasis en las citas es de los autores). En el artículo 5 (puntos 5, 6 y 7) se fijan las relaciones entre competencias y currículo a través de los ERAE que, por ello, se constituyen en los indicadores del grado de logro de las competencias:

5. Los criterios de evaluación deben servir de referencia para valorar lo que el alumnado sabe y sabe hacer en cada área o materia. Estos criterios de evaluación se desglosan en estándares de aprendizaje evaluables. Para valorar el desarrollo competencial del alumnado, serán estos estándares de aprendizaje evaluables, como elementos de mayor concreción, observables y medibles, los que, al ponerse en relación con las competencias clave, permitirán graduar el rendimiento o desempeño alcanzado en cada una de ellas.

6. El conjunto de estándares de aprendizaje evaluables de un área o materia determinada dará lugar a su perfil de área o materia.

7. Todas las áreas y materias deben contribuir al desarrollo competencial. El conjunto de estándares de aprendizaje evaluables de las diferentes áreas o materias que se relacionan con una misma competencia da lugar al perfil de esa competencia (perfil de competencia)».

El artículo 7 establece los métodos e instrumentos de evaluación de las competencias:

"Artículo 7. La evaluación de las competencias clave.

1. ... para poder evaluar las competencias es necesario elegir, siempre que sea posible, estrategias e instrumentos para evaluar al alumnado de acuerdo con sus desempeños en la resolución de problemas que simulen contextos reales, movilizando sus conocimientos, destrezas, valores y actitudes.

2. Han de establecerse las relaciones de los estándares de aprendizaje evaluables con las competencias a las que contribuyen, para lograr la evaluación de los niveles de desempeño competenciales alcanzados por el alumnado.

3. La evaluación del grado de adquisición de las competencias... supone movilizar los conocimientos, destrezas, actitudes y valores para dar respuesta a las situaciones planteadas, dotar de funcionalidad a los aprendizajes y aplicar lo que se aprende desde un planteamiento integrador. 
MARÍA ANTONIA MANASSERO-MAS Y ÁNGEL VÁZQUEZ-ALONSO

DESARROLLO CURRICULAR DE LAS COMPETENCIAS CLAVE: SU EVALUACIÓN PARA EL APRENDIZAJE DESDE LA NORMATIVA EDUCATIVA

4. Los niveles de desempeño de las competencias se podrán medir a través de indicadores de logro, tales como rúbricas o escalas de evaluación. Estos indicadores de logro deben incluir rangos dirigidos a la evaluación de desempeños, que tengan en cuenta el principio de atención a la diversidad».

En resumen, la afirmación más significativa de la norma es la necesidad de establecer las relaciones entre los estándares de aprendizaje evaluables (ERAE) y las competencias a las que estos contribuyen (una tarea de desarrollo del currículo aún pendiente). Por tanto, este mandato normativo sustenta el objetivo central de este estudio: identificar las relaciones entre los ERAE del currículo y las competencias básicas y, con ello, los perfiles competenciales de materia, que en este estudio se aplican al desarrollo curricular en algunas materias de ciencias.

\section{LA COMPETENCIA CIENTÍFICA EN PISA}

Por su impacto mundial, el programa de evaluación internacional PISA (Programme for Internacional Student Assessment) quizá sea el abanderado más conspicuo de la evaluación por competencias. En este estudio, por limitaciones de espacio y por centrar el desarrollo práctico en un área de ciencias, se analiza solo la competencia científica, también denominada cultura o alfabetización científica, como capacidad que integra actitudes, valores, conocimientos y destrezas (OCDE, 2008b). El término "alfabetización o cultura científica» en lugar de "ciencia» subraya la importancia que PISA otorga a la evaluación de la competencia científica como aplicación del conocimiento científico en el contexto de las situaciones de la vida diaria.

La definición de la cultura científica o alfabetización científica en el estudio PISA ha ido evolucionado, siguiendo los cambios producidos en la investigación didáctica sobre ese concepto y tratando de dar más coherencia al marco de evaluación de la ciencia en el estudio PISA entre 2000 y 2015. PISA 2015 la define como la habilidad (de los estudiantes) para interactuar con cuestiones relacionadas con la ciencia y con las ideas de la ciencia, como un ciudadano reflexivo, dispuesto a participar en un discurso razonado sobre la ciencia y la tecnología. A su vez, esta habilidad se desglosa en las tres competencias siguientes:

1. Explicar fenómenos científicamente. Reconocer, ofrecer y evaluar explicaciones para una serie de fenómenos naturales y tecnológicos.

2. Evaluar y diseñar la investigación científica. Describir y evaluar investigaciones científicas y proponer formas de abordar las cuestiones científicamente.

3. Interpretar datos y pruebas científicas. Analizar y evaluar los datos, las conclusiones y los argumentos en una variedad de representaciones y extraer las conclusiones científicas apropiadas.

Una persona alfabetizada científicamente no tiene suficiente con entender los conocimientos y los procedimientos que se han aplicado para obtener un conjunto 
MARÍA ANTONIA MANASSERO-MAS Y ÁNGEL VÁZQUEZ-ALONSO

DESARROLLO CURRICULAR DE LAS COMPETENCIAS CLAVE: SU EVALUACIÓN PARA EL APRENDIZAJE DESDE LA NORMATIVA EDUCATIVA

de datos. La persona alfabetizada científicamente debe ser capaz también de identificar los argumentos usados y juzgar si son apropiados para juzgar las conclusiones obtenidas (conocimiento epistémico). Por tanto, debe entender que la crítica argumentada y razonada es esencial para determinar cuál es la conclusión más apropiada; que el desacuerdo entre los científicos es más normal que extraordinario, y que la resolución sobre la mejor interpretación requiere alcanzar consensos clave acerca de ideas y procesos explicativos (Longino, 1990). De hecho, la actitud crítica y escéptica hacia todas las evidencias (pruebas) empíricas es considerada por muchos como el sello de la práctica científica profesional.

Para los fines de la evaluación de PISA 2015 (OECD, 2013) se caracteriza la alfabetización o cultura científica, además, con cuatro aspectos relacionados entre sí.

1. Contextos. Personales, locales, nacionales y globales, tanto actuales como históricos, que exigen una cierta comprensión de la ciencia y la tecnología.

2. Conocimientos. Comprensión de los principales hechos, conceptos y teorías explicativas que forman la base de los conocimientos científicos, y que incluye conceptos e ideas del mundo natural y artefactos tecnológicos (conocimiento de contenidos); los procedimientos y las estrategias utilizadas en toda forma de investigación científica que producen las ideas anteriores (conocimiento procedimental), y la comprensión de los fundamentos de esos procedimientos y de la forma en que se justifican y validan las ideas en la ciencia para su uso (conocimiento epistémico).

3. Competencias. La capacidad de explicar fenómenos científicamente; evaluar y diseñar investigación científica, e interpretar datos y evidencias (pruebas) científicas.

4. Actitudes. Un conjunto de disposiciones hacia la ciencia, cuyos indicadores son el interés hacia la ciencia y la tecnología, la valoración de los enfoques científicos apropiados de la investigación y la percepción y conciencia de los problemas ambientales.

Uno de los cambios y novedades respecto a la caracterización anterior de la competencia científica es la actual elaboración separada de los conceptos de conocimiento procedimental y conocimiento epistémico, que representan, a su vez, una especificación más detallada de algunos aspectos particulares, que estaban implícitos en las definiciones de anteriores ediciones de PISA.

\section{Metodología}

El marco jurídico legal de la evaluación de las competencias marca unos principios normativos claros: para valorar el desarrollo competencial del alumnado, los ERAE son los elementos curriculares de mayor concreción, observables y medibles que la normativa establece como referencias básicas de las competencias clave, y que permitirán evaluar el desempeño competencial alcanzado en cada una de 

DESDE LA NORMATIVA EDUCATIVA

las materias. Puesto que el currículo normativo prescrito no desarrolla la relación ERAE-competencias, una adecuada planificación de su enseñanza y una evaluación orientada al aprendizaje de las competencias pasa por identificar las relaciones de los ERAE disciplinares con las competencias a las que contribuyen (perfil competencial de la materia), como indicadores de la evaluación competencial y, por tanto, como paso previo para lograr la evaluación de los niveles competenciales alcanzados por el alumnado y fundamentar mejor su enseñanza y aprendizaje (evaluación para el aprendizaje).

\section{INSTRUMENTO}

Los límites de extensión obligan a reducir el análisis al currículo de una materia concreta, que en este caso será la Física y Química de segundo y tercero de educación secundaria obligatoria -ESO- (análisis de caso). Como enfoque tácito del análisis se supone que todos los ERAE del currículo de esta materia se refieren ya a la competencia básica en ciencia y tecnología (свсуT), de modo que, para no extender estas repeticiones obvias, se acepta axiomáticamente este supuesto y ya no se explicita. Por ello, el análisis se centra en la contribución de los ERAE al desarrollo de las otras competencias distintas de la свсут, porque desde cada materia el problema relevante es identificar sus aportaciones específicas a todas las competencias, para conseguir aprendizajes realmente competenciales, acreditados por su integración, transversalidad y transferibilidad.

\section{PROCEDIMIENTO}

La metodología de análisis del contenido se aplica a los ERAE del currículo, en cumplimiento de la norma, para demostrar la relación entre currículo y competencias, construir el perfil competencial de la asignatura analizada y demostrar empíricamente la contribución de esta asignatura al desarrollo de todas las competencias. El análisis de contenido realizado tratará de identificar aquellos ERAE del currículo de la materia científica elegida que contribuyen al desarrollo de las diferentes competencias. En este proceso se tienen en cuenta algunos criterios. Cuando la redacción de un ERAE es tan concreta que solo se puede corresponder con algún componente singular de la competencia científica (conocimiento, proceso, actitud, etc.), pero no refleja la integración de saberes necesaria para considerarse un indicador competencial, este ERAE se excluye del análisis para la evaluación de competencias y no se explicita en la tabla de resultados, para permitir una mayor focalización del análisis en los ERAE del currículo realmente relacionados con un nivel de competencia (integración de saberes) y aliviar de información superflua los perfiles competenciales (por el límite de extensión).

En la mayoría de casos, los verbos de acción descriptores de los ERAE se corresponden mayoritariamente con las categorías cognitivas de la conocida taxonomía 
MARÍA ANTONIA MANASSERO-MAS Y ÁNGEL VÁZQUEZ-ALONSO

DESARROLLO CURRICULAR DE LAS COMPETENCIAS CLAVE: SU EVALUACIÓN PARA EL APRENDIZAJE DESDE LA NORMATIVA EDUCATIVA

de Bloom (conocer, describir, relacionar, realizar, distinguir, comprobar, relacionar... conceptos y procesos... etc.). Sin embargo, a pesar de la especificidad postulada por el legislador para los ERAE, la redacción de estos es con frecuencia ambigua o múltiple; en estos casos, los ERAE pueden corresponderse con más de una competencia simultáneamente y entonces el ERAE se atribuye a todas las competencias que resulten compatibles con su ambigua redacción.

En síntesis, las preguntas de investigación que pretende responder este estudio son las siguientes:

¿Cuál es el perfil competencial de la asignatura de Física y Química de EsO?

¿Cuál es la contribución de la asignatura Física y Química de ESO a las otras competencias diferentes de la competencia básica en ciencia y tecnología (свсут)?

¿Cómo se podrían evaluar los ERAE correspondientes a las distintas competencias y a la CBCyT?

\section{Resultados}

Los resultados básicos de este estudio están formados por las relaciones identificadas entre las competencias básicas y los elementos del currículo. Como se ha mencionado anteriormente, los ERAE son considerados por la normativa como los indicadores de logro de las competencias básicas y, por ello, constituyen la referencia obligada más concreta del currículo. Por tanto, los resultados obtenidos son consecuencia del análisis del contenido de los ERAE para ponerlos en relación con las competencias básicas a las cuales correspondan, en la materia elegida para desarrollar esta ejemplificación (Física y Química de ESO).

Los resultados del análisis de contenido de los ERAE de los distintos bloques de contenidos del currículo para establecer su correspondencia con competencias para la materia mencionada forman el perfil competencial de la materia. La Tabla 2 presenta el perfil competencial del primer bloque de la asignatura, referido a la actividad científica, como ejemplo reducido del trabajo. Los resultados muestran una contribución relevante de este bloque a todas las competencias, pues todas las competencias tienen algún ERAE asignado como indicador evaluable. Los ERAE 1.2 y 6.1 son un ejemplo de indicadores cuyo contenido es transversal a dos competencias; por ejemplo, el ERAE 6.1 posee contenidos de la competencia aprender a aprender y de la competencia digital. 
MARÍA ANTONIA MANASSERO-MAS Y ÁNGEL VÁZQUEZ-ALONSO

DESARROLLO CURRICULAR DE LAS COMPETENCIAS CLAVE: SU EVALUACIÓN PARA EL APRENDIZAJE DESDE LA NORMATIVA EDUCATIVA

TABLA 2

Perfil competencial para el bloque 1 del currículo de la asignatura Física y Química (cursos segundo y tercero de ESO)

COMPETENCIAS
CLAVE

1.2. Registra observaciones, datos y resultados de manera organizada y rigurosa, y los comunica de forma oral y escrita utilizando esquemas, Lingüística gráficos, tablas y expresiones matemáticas.

5.1. Selecciona, comprende e interpreta información relevante en un texto de divulgación científica y transmite las conclusiones obtenidas utilizando el lenguaje oral y escrito con propiedad.

1.2. Registra observaciones, datos y resultados de manera organizada y

Matemática,
ciencia y
tecnología

Digital

Aprender a aprender

Sociales y cívicas rigurosa, y los comunica de forma oral y escrita utilizando esquemas, gráficos, tablas y expresiones matemáticas.

3.1. Establece relaciones entre magnitudes y unidades utilizando, preferentemente, el Sistema Internacional de Unidades y la notación científica para expresar los resultados.

5.2. Identifica las principales características ligadas a la fiabilidad y objetividad del flujo de información existente en internet y otros medios digitales.

6.1. Realiza pequeños trabajos de investigación sobre algún tema objeto de estudio aplicando el método científico, y utilizando las TIC para la búsqueda y selección de información y presentación de conclusiones.

6.1. Realiza pequeños trabajos de investigación sobre algún tema objeto de estudio aplicando el método científico, y utilizando las TIC para la búsqueda y selección de información y presentación de conclusiones.

4.1. Reconoce e identifica los símbolos más frecuentes utilizados en el etiquetado de productos químicos e instalaciones, interpretando su significado.

4.2. Identifica material e instrumentos básicos de laboratorio y conoce su forma de utilización para la realización de experiencias respetando las normas de seguridad e identificando actitudes y medidas de actuación preventivas.

6.2. Participa, valora, gestiona y respeta el trabajo individual y en equipo.

Iniciativa y 1.1. Formula hipótesis para explicar fenómenos cotidianos utilizando emprendedor teorías y modelos científicos.

Cultural 2.1. Relaciona la investigación científica con las aplicaciones tecnológicas en la vida cotidiana. 
MARÍA ANTONIA MANASSERO-MAS Y ÁNGEL VÁZQUEZ-ALONSO DESDE LA NORMATIVA EDUCATIVA

Por brevedad, se han omitido aquellos ERAE que contribuyen de manera autoevidente a la competencia básica en matemáticas, ciencia y tecnología (cuya identificación no supone ningún problema semántico) y aquellos otros cuya formulación no cumple la definición de competencia (integración de conocimiento, destrezas y actitudes), aunque todos ellos serían indicadores para la evaluación de la свСут.

De forma análoga, se desarrollan los perfiles competenciales de los otros bloques de contenidos de la asignatura elegida, sobre los que solo se comentan algunos rasgos destacados. El perfil competencial del bloque 2 (la materia) también se extiende sobre la mayoría de competencias básicas, con excepción de la competencia social y cívica.

El perfil competencial del bloque de contenidos referido a los cambios carece de ERAE para las competencias lingüística y digital (ya no se menciona el caso de su obvia contribución a la СвСут), pero aporta una buena representación sobre las otras competencias restantes.

El perfil competencial del bloque sobre movimiento y fuerzas destaca por la cantidad de ERAE relacionados con el desarrollo de la competencia digital, aunque tres competencias (lingüística, social y emprendedora) carecen de ERAE relacionados que las desarrollen desde este bloque.

Globalmente, el perfil competencial de la asignatura Física y Química de segundo y tercero de ESO encontrado indica que el currículo de esta asignatura puede contribuir significativamente al desarrollo de otras competencias diferentes de la propia свсут (objeto principal del currículo de esta asignatura) y concretando los indicadores ERAE que los relacionan. La competencia lingüística se desarrolla a través de tres ERAE; la competencia digital tiene cinco ERAE que la desarrollan; análogamente, la competencia de aprender a aprender es desarrollada por cuatro ERAE del currículo; la competencia social y cívica es desarrollada por cinco ERAE del currículo; la competencia de iniciativa y emprendedor es desarrollada por cuatro ERAE del currículo, y la competencia cultural es desarrollada por cinco ERAE del currículo. Así pues, en conjunto, el análisis del perfil competencial permite concluir que el currículo de la asignatura contribuye significativamente a las otras competencias diferentes de свСут pues el análisis de contenido ha encontrado un conjunto de indicadores suficientes para la evaluación de las competencias desde esta asignatura.

Los resultados anteriores identifican los indicadores básicos para la evaluación de las competencias en una asignatura específica. Esta identificación resuelve el problema, relevante para el profesorado, de concretar cómo su asignatura puede contribuir transversalmente al desarrollo de otras competencias, que aparentemente difieren ampliamente en los contenidos curriculares de la asignatura. Estos indicadores identificados en la forma de ERAE definen el objeto de la evaluación competencial; por tanto, para su implementación en la evaluación competencial, el profesorado debe elegir los instrumentos apropiados para recoger los datos y las evidencias que le permitan valorar el grado de consecución de cada uno de ellos. 

DESDE LA NORMATIVA EDUCATIVA

En la introducción se mencionaron algunos instrumentos aconsejables (rúbricas, portafolios, observación, escalas, listas, etc.) para evaluar estos indicadores competenciales, porque muy frecuentemente requieren valorar algún tipo de ejecución de los alumnos. Como ejemplo de estos instrumentos, se desarrolla una rúbrica sencilla para evaluar un indicador muy común y extendido en muchas asignaturas como es la realización de trabajos de investigación («6.1. Realiza pequeños trabajos de investigación sobre algún tema objeto de estudio aplicando el método científico, y utilizando las TIC para la búsqueda y selección de información y presentación de conclusiones»), un ERAE que también contribuye a la competencia digital y a aprender a aprender (Tabla 3).

La rúbrica contempla la evaluación de los tres elementos descritos en el ERAE, a saber, el trabajo de investigación, la aplicación del método científico en ese trabajo y el uso de las TIC para buscar y seleccionar información y presentar el trabajo. Cada uno de estos elementos básicos del trabajo se desarrolla en la rúbrica mediante una serie de criterios de evaluación más específicos. El trabajo se valora con criterios de contenido (planteamiento, justificación, contenidos) y de forma (organización, redacción, vocabulario, ortografía y formatos). La aplicación del método científico se valora con criterios sobre la calidad de las hipótesis emitidas; de la toma de datos, y de la fundamentación, argumentación y conclusiones del trabajo. La aplicación de Tic tiene en cuenta los criterios de búsqueda de información, selección de la información e implementación de Tic en la presentación del trabajo.

TABLA 3

Rúbrica para evaluar el estándar y resultado de aprendizaje evaluable: realiza pequeños trabajos de investigación (6.1)*

\begin{tabular}{|l|l|l|l|l|l|}
\hline & & & & \\
\hline $\begin{array}{l}\text { Planteamiento } \\
\text { del tema } \\
\text { del trabajo }\end{array}$ & $\begin{array}{l}\text { el tema en } \\
\text { la primera } \\
\text { frase de cada } \\
\text { párrafo y en } \\
\text { el cuerpo }\end{array}$ & $\begin{array}{l}\text { Plantean } \\
\text { claramente } \\
\text { el tema en } \\
\text { el cuerpo }\end{array}$ & $\begin{array}{l}\text { el tema en } \\
\text { poca claridad } \\
\text { las primeras } \\
\text { frases }\end{array}$ & $\begin{array}{l}\text { poca claridad } \\
\text { el tema en } \\
\text { las primeras } \\
\text { frases y en } \\
\text { el cuerpo }\end{array}$ & $\begin{array}{l}\text { No plantean } \\
\text { claramente } \\
\text { el tema ni en } \\
\text { la primera } \\
\text { frase ni en } \\
\text { el cuerpo }\end{array}$ \\
\hline $\begin{array}{l}\text { Justificación } \\
\text { del trabajo }\end{array}$ & $\begin{array}{l}\text { Muchos } \\
\text { detalles y } \\
\text { razones }\end{array}$ & $\begin{array}{l}\text { Varios } \\
\text { detalles o } \\
\text { razones }\end{array}$ & $\begin{array}{l}\text { Algún detalle } \\
\text { o razón }\end{array}$ & $\begin{array}{l}\text { Un detalle } \\
\text { o razón }\end{array}$ & $\begin{array}{l}\text { Ningún } \\
\text { detalle o } \\
\text { razón }\end{array}$ \\
\hline
\end{tabular}



DESDE LA NORMATIVA EDUCATIVA

\begin{tabular}{|l|l|l|l|l|l|}
\hline & & & & \\
\hline $\begin{array}{l}\text { Contenido } \\
\text { del trabajo }\end{array}$ & $\begin{array}{l}\text { Enfocado, } \\
\text { detallado, } \\
\text { profundo, } \\
\text { preciso }\end{array}$ & $\begin{array}{l}\text { Enfocado } \\
\text { y preciso, } \\
\text { pero poco } \\
\text { detallado, o } \\
\text { profundo }\end{array}$ & $\begin{array}{l}\text { Enfocado, } \\
\text { pero poco } \\
\text { detallado o } \\
\text { profundo } \\
\text { o preciso }\end{array}$ & Enfocado & Desenfocado \\
\hline $\begin{array}{l}\text { Organización } \\
\text { del trabajo }\end{array}$ & $\begin{array}{l}\text { Todos los } \\
\text { apartados } \\
\text { necesarios; } \\
\text { sucesión } \\
\text { lógica y } \\
\text { transiciones } \\
\text { excelentes } \\
\text { entre } \\
\text { apartados; } \\
\text { conclusión } \\
\text { importante }\end{array}$ & $\begin{array}{l}\text { Todos los } \\
\text { apartados } \\
\text { necesarios; } \\
\text { sucesión } \\
\text { lógica de } \\
\text { apartados; } \\
\text { conclusión } \\
\text { importante }\end{array}$ & $\begin{array}{l}\text { Todos los } \\
\text { apartados } \\
\text { necesarios; } \\
\text { sucesión } \\
\text { lógica de } \\
\text { apartados }\end{array}$ & $\begin{array}{l}\text { Faltan } \\
\text { apartados } \\
\text { necesarios } \\
\text { o sucesión } \\
\text { de apartados } \\
\text { no lógica }\end{array}$ & $\begin{array}{l}\text { Faltan } \\
\text { apartados } \\
\text { necesarios y } \\
\text { sucesión de } \\
\text { apartados } \\
\text { no lógica }\end{array}$ \\
\hline
\end{tabular}

* Esta rúbrica con solo cuatro criterios es incompleta para ajustarse a las limitaciones de espacio (la versión completa tiene veinte criterios).

\section{CONCLUSIONES Y DISCUSIÓN}

La evaluación de las competencias clave en los aprendizajes educativos constituye un problema relevante para la práctica educativa (por el imperativo normativo existente de enseñar y evaluar por competencias) y para la investigación (por la necesidad de coherencia y calidad entre currículo y evaluación). El ejemplo más paradigmático de la relevancia de la evaluación para el aprendizaje de las competencias es el cambio y la evolución experimentados por los marcos teóricos de evaluación del proyecto PISA desde sus inicios en el año 2000 hasta hoy, asistido por los mejores especialistas en investigación educativa (OECD, 2015). Obviamente, para un profesor individual la dificultad de desarrollar el currículo enseñado con base en las competencias puede ser un grave escollo por su eventual falta de formación, experiencia, recursos y apoyo y la tradicional aridez del lenguaje educativo normativo. Este estudio ofrece una guía, para ayudar a superar esta dificultad a través del desarrollo del currículo con base en las competencias clave, atendiendo a la normativa educativa que desarrolla las competencias clave y los currículos basados en los estándares y resultados de aprendizaje evaluables (ERAE) mediante un ejercicio de correspondencias en una asignatura concreta. 

DESDE LA NORMATIVA EDUCATIVA

Este estudio aplica la normativa del desarrollo de las competencias clave con base en los ERAE al currículo de la asignatura de Física y Química de segundo y tercero de EsO. Además, se desarrolla también un instrumento ejemplar de rúbrica de evaluación para valorar uno de los ERAE del currículo de esa asignatura que desarrolla competencias y, por tanto, se convierte en un instrumento de evaluación de competencias. Ambos elementos ejemplifican instrumentos, pistas y orientaciones concretas para ayudar al profesorado a afrontar el desarrollo de sus currículos con base en las competencias básicas, siguiendo las pautas ofrecidas.

LOS ERAE que desarrollan la СВCуT para la asignatura elegida ya no se incluyen en el desarrollo de las competencias, pues su inclusión, al tratarse de una asignatura de ciencias, supondría una mera redundancia obvia, pues aportaría poco al profesorado y complicaría innecesariamente la presentación de los resultados (además de ajustar el texto a la restricción de espacio). Otra limitación de este estudio derivada del espacio limitado es que el currículo ejemplificado puede ser más profundizado y personalizado aún más por cada profesor, por ejemplo, concretando mucho más los ERAE a través del desarrollo de los instrumentos de evaluación personalizados por cada profesor, por ejemplo, elaborando rúbricas similares a la presentada en este estudio.

No obstante, en los últimos años las competencias están siendo reorientadas hacia enfoques aún más holísticos e interdisciplinares a través de las denominadas destrezas transversales mencionadas (Unión Europea, 2006) debido a la acelerada evolución del mercado laboral del siglo xxI. Las demandas de competencias se centran en una capacidad global de aplicación de conocimientos, habilidades y responsabilidades a nuevos entornos y problemas complejos; entre las habilidades, los expertos están abrumadoramente de acuerdo en señalar como esenciales el pensamiento crítico y creativo, la comunicación escrita y oral, la investigación y el análisis y el trabajo en equipo. Este nuevo marco es una tendencia crecientemente consensuada por diversos actores sociales y que claramente desborda el anterior marco teórico de varias competencias básicas; el sistema educativo deberá tener en cuenta esta nueva orientación hacia una mayor globalidad, integración e interdisciplinariedad en la definición de una única competencia global, que constituyen nuevos factores del futuro (ver https://www.aacu.org/sites/default/files/files/ LEAP/2018EmployerResearchReport.pdf).

En suma, la ejemplificación presentada en este estudio puede contribuir a facilitar la evaluación de las competencias clave con base en el currículo de cada asignatura en la práctica del aula, fijando la contribución de cada asignatura al desarrollo de todas las competencias claves a través de su perfil competencial basado en los ERAE del currículo y a preparar un futuro de las competencias aún más transversal, global, integrado e interdisciplinar. 
MARÍA ANTONIA MANASSERO-MAS Y ÁNGEL VÁZQUEZ-ALONSO

DESARROLLO CURRICULAR DE LAS COMPETENCIAS CLAVE: SU EVALUACIÓN PARA EL APRENDIZAJE DESDE LA NORMATIVA EDUCATIVA

\section{REFERENCIAS BIBLIOGRÁFICAS}

Bolívar, A. (2008, junio). El discurso de las competencias en España: educación básica y educación superior. Red U. Revista de Docencia Universitaria, número monográfico I1 Formación centrada en competencias (II). http://www.redu.m.es/Red_U/m2.

Cano García, M. E. (2008). La evaluación por competencias en la educación superior. Profesorado. Revista de Currículum y Formación del Profesorado, 12, 3. http://www.ugr.es/ local/recfpro/rev123col1.pdf.

Cañas, A.; Martín, M. J. y Nieda, J. (2007). Competencia en el conocimiento y la interacción con el mundo físico. La competencia científica. Madrid: Alianza Editorial.

Delors, J. (Pte.) (1996). La educación encierra un tesoro. Informe a la UNESCO de la Comisión internacional sobre la educación para el siglo XXI. Madrid: Santillana/UNESCO.

EURYDICE (2002). Las Competencias Clave. http://comclave.educarex.es/pluginfile.php/126/ mod_resource/content/2/Competencias_clave_Eurydice.pdf (20.XII.2014).

Fisher, A. (2009). Critical Thinking. An Introduction. Cambridge: Cambridge U. Press.

Longino, H. E. (1990). Science as social knowledge: Values and objectivity in scientific inquiry. Princeton, NJ: Princeton University Press.

Looney, J. y Michel, A. (2014). Keyconet's conclusions and recommendations for strengthening key competence development in policy and practice. Final report. Brussels: European Schoolnet. http://keyconet.eun.org/c/document_library/get_file?uuid=78469b98-b49c4e9a-a1ce-501199f7e8b3\&groupId=11028.

Marina, J. A. (2010). La Educación del Talento. Barcelona: Editorial Ariel.

Manassero-Mas, M. A. y Vázquez-Alonso, A. (2019). Taxonomía de las destrezas de pensamiento: una herramienta clave para la alfabetización científica. En M. D. Maciel y E. Albrecht (org.), Ciência, Tecnologia \& Sociedade: Ensino, Pesquisa e Formação (pp. 17-38). Sao Paulo: UNICSUL.

Ministerio de Educación, Cultura y Deporte. (2013). Ley Orgánica 8/2013, de 9 de diciembre, para la mejora de la calidad educativa. $B O E$ N. ${ }^{\circ}$ 295, 10/12/2013.

Ministerio de Educación, Cultura y Deporte. (2015). Orden ECD/65/2015, de 21 de enero, por la que se describen las relaciones entre las competencias, los contenidos y los criterios de evaluación de la educación primaria, la educación secundaria obligatoria y el bachillerato. BOE núm. 25, de 29 de enero de 2015, 6986-7003.

Monarca, H. y Rappoport, S. (2013). Investigación sobre los procesos de cambio educativo: El caso de las competencias básicas en España. Revista de Educación, número extraordinario, Competencias básicas: retórica o realidad. https://doi.org/10.4438/1988-592XRE-2013-EXT-256.

OECD. (2002). Definition and Selection of Competencies (DeSeCo), Theoretical and Conceptual Foundations (Strategy Paper). http://www.deseco.admin.ch/bfs/deseco/en/ index/02.parsys.34116.downloadList.87902.DownloadFile.tmp/oecddesecostrategypaperdeelsaedcericd20029.pdf (28.XII.2014).

OECD. (2008a). Assessment for Learning Formative Assessment. OECD/CERI International Conference Learning in the 21st Century: Research, Innovation and Policy. https://www. oecd.org/site/educeri21st/40600533.pdf.

OECDE. (2008b). Informe PISA 2006. Competencias científicas para el mundo del mañana. Madrid: Santillana Educación.

OECD. (2013). PISA 2015. Draft science framework. Paris: http://www.oecd.org/pisa/pisaproducts/Draft\%20PISA\%202015\%20Science\%20Framework\%20.pdf 
Pereda, S. y Berrocal, F. (2001). Técnicas de gestión de recursos bumanos por competencias. Madrid: Centro de Estudios Ramón Areces.

Unión Europea. (2006). Recomendaciones del Parlamento Europeo y del Consejo, de 18 de diciembre de 2006 sobre las competencias clave para el aprendizaje permanente (2006/962/CE). Diario Oficial de la Unión Europea, 30/12/2006.

Valle, J. y Manso, J. (2013). Competencias clave como tendencia de la política educativa supranacional de la Unión Europea. Revista de Educación, número extraordinario, Competencias básicas: retórica o realidad. https://doi.org/10.4438/1988-592X-RE-2013EXT-255

VV. AA. (2013). Competencias básicas: retórica y realidad. Revista de Educación, número extraordinario. Madrid: MECD. 